
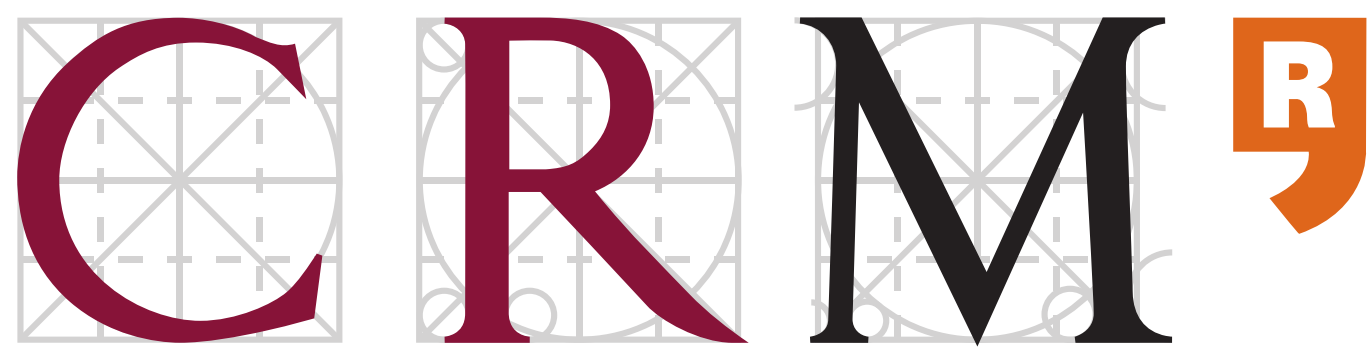

CENTRE DE RECERCA MATEMÀTICA

This is a preprint of: Some inequalities of trigonometric approximation in weighted Orlicz spaces

Journal Information: CRM Preprints,

Author(s):

RAMAZAN AKGÜN.

Volume, pages:

$1-21$,

DOI:[--] 


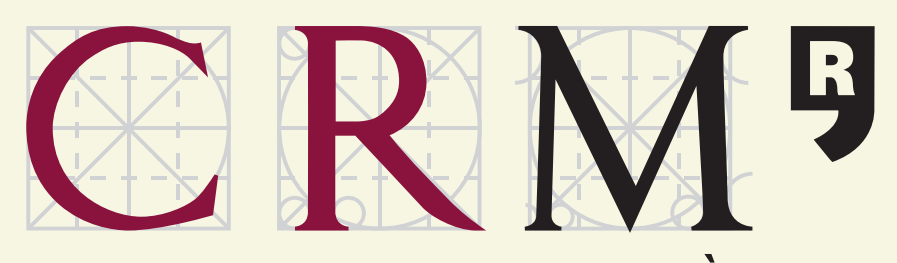

\section{CENTRE DE RECERCA MATEMÀTICA}

Preprint núm. 1172

September 2013

Some inequalities of trigonometric approximation in weighted Orlicz spaces

R. Akgün 



\title{
SOME INEQUALITIES OF TRIGONOMETRIC APPROXIMATION IN WEIGHTED ORLICZ SPACES
}

\author{
RAMAZAN AKGÜN
}

\begin{abstract}
In the present work, we proved a refined direct theorem and an exact inverse theorem of trigonometric approximation in Orlicz spaces with weights satisfying some Muckenhoupt's $A_{p}$ condition. As a consequence, refined Marchaud inequality and its inverse are obtained.
\end{abstract}

\section{INTRODUCTION AND THE MAIN RESULTS}

For trigonometric approximation of functions in Lebesgue spaces with Muckenhoupt weights, the first results appeared in the $\mathrm{PhD}$ thesis [Gadj] of E. A. Gadjieva. Since the space is not necessarily translation invariant she defined a weighted modulus of smoothness (see definition of $\Omega_{r}$ below) and proved the Jackson type direct theorem and an inverse theorem of trigonometric approximation. Later inverse approximation theorems of Gadjieva is improved in [GI]. Recently, author improved the Jackson inequality of Gadjieva (see [A]). On the other hand a different method of trigonometric approximation in Lebesgue spaces with Muckenhoupt weights, developed by N. X. Ky ([Ky1] and [Ky2]). He defined also another weighted modulus of smoothness (see definition of $\bar{\Omega}_{r}$ below). Independently of Gadjieva, Ky proved the direct and inverse theorems of trigonometric approximation in Lebesgue spaces with Muckenhoupt weights. We know that these modulus of smoothnesses are equivalent for $r \in \mathbb{N}$, namely, $\bar{\Omega}_{2 r} \sim \Omega_{r}$. Ky's inverse theorem is improved in [YI]. For the more general Orlicz spaces, similar problems are investigated for Muckenhoupt weights. The direct and inverse theorems of trigonometric approximation (with Gadjieva modulus) in Orlicz spaces with Muckenhoupt weights were proved in [IG] (weight is inside and $M$ is convex Young function) and [AI1] (weight is outside and $M$ is quasiconvex Young function). Also some inverse theorems of trigonometric approximation of functions and its fractional derivatives in Orlicz spaces with Muckenhoupt weights were proved in $[\mathrm{AI}]$. On the other hand, for the Orlicz spaces, there is ([Che1]) a

2010 Mathematics Subject Classification: Primary 41A10, 42A10; Secondary 26A33, 41A17, $41 \mathrm{~A} 25,41 \mathrm{~A} 27$.

Key words and phrases: weighted fractional modulus of smoothness, direct theorem, inverse theorem, fractional derivative, Orlicz space, trigonometric polynomial approximation.

Partially supported by grant 2219 no. 2012-1-9246 of The Scientific and Technological Research Council of Turkey, TÜBITAK, MTM2011-27637. 
different approach. In [Che1], Chen is generalized the definition of Orlicz spaces saving almost all known properties of them. In this work we will develop this approach with Muckenhoupt weights and prove refined Jackson theorem and exact inverse theorem of trigonometric approximation in these spaces. Let us note required definitions and notations. Let $\Phi$ be the class of strictly increasing functions $\phi:[0, \infty) \rightarrow[0, \infty)$ satisfying $\phi(\infty):=\lim _{x \rightarrow \infty} \phi(x)=\infty$. Let $-\infty<p \leq q<\infty$. By $Y[p, q]$ we denote the class of even functions $\varphi \in \Phi$ satisfying

(i) $\varphi(u) / u^{p}$ is non-decreasing as $|u|$ increases;

(ii) $\varphi(u) / u^{q}$ is non-increasing as $|u|$ increases.

If $p<q$, the class of functions $\varphi$ belonging to $Y[p+\varepsilon, q-\delta]$ for some small numbers $\varepsilon, \delta>0$ will be denote by $Y\langle p, q\rangle$. By $\bar{\Phi}_{p}$ we will denote the class of functions $M$ such that $M$ belongs to the class $Y\langle p, q\rangle$ for some $1<p \leq q<\infty$. Every function $M$ of class $\bar{\Phi}_{p}, p>1$, is continuous and satisfy $M(0)=0$ and $\Delta_{2}$ condition (in short $M \in \Delta_{2}$ ), that is, there is a constant $C>0$ and $x_{0}>0$ such that $M(2 x) \leq C M(x)$ for all $x \geq x_{0}$. The condition $M \in \bar{\Phi}_{p}, p>1$, need not imply $M$ to be convex. (See the example given in [Che1, pp:67-68]). Throughout this work, by $C(\ldots)$, we denote constants which are different in different places and depends only on the essential parameters given in the bracket. Two functions $f_{1}, f_{2}$ of class $\bar{\Phi}_{p}, p>1$, are said to be equivalent (in short $f_{1} \sim f_{2}$ ) if there are constants $C, C^{\prime}>0$ such that $f_{2}(C x) \leq f_{1}(x) \leq f_{2}\left(C^{\prime} x\right)$, for all $x>0$. A function $M$ of class $\Phi$, satisfying $M(0)=0$, is said to be quasiconvex ([KK]) if there exist a convex function $\phi$ and a constant $C \geq 1$ such that $\phi(x) \leq M(x) \leq$ $\phi(C x)$ for every $x \geq 0$. Hence every convex function $M$ is quasiconvex. A nonnegative function $\omega$ defined on $\mathbb{T}:=[0,2 \pi]$ will be called a weight if $\omega$ is measurable and positive almost everywhere (a.e.). Let $M \in \bar{\Phi}_{p}, p>1$, and $\omega$ be weight on $\mathbb{T}$. We denote $\varphi_{M}(t)=M(t) / t$. Since $1<p<q<\infty$, we have $\varphi_{M}(t) \rightarrow \infty$ as $t \rightarrow \infty$. Let $\psi_{M}(t)$ be the inverse function of positive nondecreasing continuous function $\varphi_{M}(t)$. We write

$$
\Phi_{M}(x)=\int_{0}^{x} \varphi_{M}(t) d t \text { and } \Psi_{M}(x)=\int_{0}^{x} \psi_{M}(t) d t .
$$

The function $\Phi_{M}$ is a convex and hence $\Phi_{M}$ and $\Psi_{M}$ are complementary functions in the sense of Young. We denote by $L_{M, \omega}(\mathbb{T})$, the class of Lebesgue measurable functions $f: \mathbb{T} \rightarrow \mathbb{R}$ satisfying the condition

$$
\int_{\mathbb{T}} \Phi_{M}(C|f(x)|) \omega(x) d x<\infty
$$

for some positive real constant $C$. We set

$$
\|f\|_{[M], \omega}:=\sup _{g}\left\{\int_{\mathbb{T}}|f(x) g(x)| \omega(x) d x: \int_{\mathbb{T}} \Psi_{M}(|g(x)|) \omega(x) d x \leq 1\right\},
$$




$$
\|f\|_{\langle M\rangle, \omega}:=\inf \left\{\tau>0: \int_{\mathbb{T}} \Phi_{M}\left(\frac{|f(x)|}{\tau}\right) \omega(x) d x \leq 1\right\} .
$$

In this case, we have the equivalence

$$
\|f\|_{\langle M\rangle, \omega} \sim\|f\|_{[M], \omega} .
$$

It can be easily seen that $L_{M, \omega}(\mathbb{T}) \subset L^{1}(\mathbb{T})$ and $L_{M, \omega}(\mathbb{T})$ becomes a Banach space with the above norms. The Banach space $L_{M, \omega}(\mathbb{T})$ is called weighted Orlicz space. For a weight $\omega$, taking $M(x, p):=x^{p}, 1<p<\infty$, we denote $L^{p}(\mathbb{T}, \omega):=L_{M(\cdot, p), \omega}(\mathbb{T})$ the weighted Lebesgue space.

A $2 \pi$-periodic weight function $\omega$ belongs to the Muckenhoupt class $A_{p}, 1 \leq$ $p<\infty$, if

$$
\left(\frac{1}{|J|} \int_{J} \omega(x) d x\right)\left(\frac{1}{|J|} \int_{J} \omega^{\frac{1}{1-p}}(x) d x\right)^{p} \leq C \text { for } 1<p<\infty,
$$

and

$$
\frac{1}{|J|} \int_{J} \omega(x) d x \leq C \operatorname{essinf} \omega(x) \leq C \omega(x) \text { for } p=1,
$$

with a finite constant $C$ independent of $J$, where $J$ is any subinterval of $\mathbb{T}$ and $|J|$ denotes the length of $J$.

Let $A_{\infty}:=\cup_{p \geq 1} A_{p}$. The following Extrapolation theorem is useful for our considerations:

Theorem 1. [CGMP, Theorem 3.1] Let $0<p_{0}<\infty$ and let $\mathcal{F}$ be a family of couples of nonnegative functions such that

$$
\int_{\mathbb{T}} f(x)^{p_{0}} \omega(x) d x \leq C \int_{\mathbb{T}} g(x)^{p_{0}} \omega(x) d x, \quad(f, g) \in \mathcal{F}
$$

I for all $\omega \in A_{\infty}$ provided the left hand side is finite. Let $\phi \in \Phi$ such that

(i) $\phi \in \Delta_{2}$ and $M(0)=0$

(ii) there exist some exponents $0<r_{0}, s_{0}<\infty$ such that $\phi\left(x^{r_{0}}\right)^{s_{0}}$ is quasiconvex. Then for all $\omega \in A_{\infty}$ the inequality

$$
\int_{\mathbb{T}} \phi(f(x)) \omega(x) d x \leq C \int_{\mathbb{T}} \phi(g(x)) \omega(x) d x
$$

holds for any $(f, g) \in \mathcal{F}$ when the left-hand side is finite.

Remark 1. If $M \in \bar{\Phi}_{p}, 1<p<q<\infty$, then [Che1, Lemma 3] $p \Phi_{1}(x) \leq$ $M(x) \leq q \Phi_{1}(x)$ and therefore $M$ is quasiconvex. Also we have $M \sim \Phi_{1}$. Therfore from (1.2) we have $\|f\|_{\langle M\rangle, \omega} \leq C\|g\|_{\langle M\rangle, \omega}$ provided (1.1) holds. 
Definition 1. A normed linear space $X$ is said to be q-concave if $0 \leq f_{i} \in X$, $i=1,2,3, \ldots, n \in \mathbb{N}$, the inequality

$$
\left\{\sum_{i=1}^{n}\left\|f_{i}\right\|_{X}^{q}\right\}^{1 / q} \leq C\left\|\left(\sum_{i=1}^{n} f_{i}^{q}\right)^{1 / q}\right\|_{X}
$$

holds. $X$ is said to be p-convex if

$$
\left\{\sum_{i=1}^{n}\left\|f_{i}\right\|_{X}^{p}\right\}^{1 / p} \geq C\left\|\left(\sum_{i=1}^{n} f_{i}^{p}\right)^{1 / p}\right\|_{X}
$$

holds.

If $M \in \bar{\Phi}_{p}, p>1$, then considering Corollary 3.4 and Theorem 5.1 of [HKT] we get that $M\left(x^{1 /(p+\varepsilon)}\right)$ is convex (hence it is quasiconvex). Therefore using Extrapolation Theorem 1 and Remark 1, it is easy to see that, if $M \in \bar{\Phi}_{p}, p>1$, and $\omega \in A_{p}$, then the Hardy-Littlewood Maximal operator is norm bounded in $L_{M, \omega}(\mathbb{T})$.

Let $x, t \in \mathbb{R}, \alpha \in \mathbb{R}^{+}:=(0, \infty)$ and we set

$$
\Delta_{t}^{\alpha} f(x):=\sum_{k=0}^{\infty}(-1)^{\alpha}\left[C_{k}^{\alpha}\right] f(x+(\alpha-k) t), \quad f \in L_{M, \omega}(\mathbb{T}),
$$

where $\left[C_{k}^{\alpha}\right]:=\frac{\alpha(\alpha-1) \ldots(\alpha-k+1)}{k !}$ for $k \geq 1$ and $\left[C_{0}^{\alpha}\right]:=1$. Since $\left|\left[C_{k}^{\alpha}\right]\right| \leq \frac{C(\alpha)}{k^{\alpha+1}}, k \in \mathbb{N}$, we have $\sum_{k=0}^{\infty}\left|\left[C_{k}^{\alpha}\right]\right|<\infty$ and $\Delta_{t}^{\alpha} f$ is defined a.e. If $\alpha \in \mathbb{N}$, then the fractional difference $\Delta_{t}^{\alpha} f$ coincides with usual forward difference, namely,

$$
\Delta_{t}^{\alpha} f(x)=\sum_{k=0}^{\alpha}(-1)^{\alpha}\left[C_{k}^{\alpha}\right] f(x+(\alpha-k) t)=\sum_{k=0}^{\infty}(-1)^{\alpha-k}\left[C_{k}^{\alpha}\right] f(x+k t)
$$

for $\alpha \in \mathbb{N}$ and $x \in \mathbb{T}$.

Taking $f \in L_{M, \omega}(\mathbb{T})$ we define means

$$
\mathcal{A}_{h} f(x):=\frac{1}{h} \int_{x-h / 2}^{x+h / 2} f(t) d t, \quad \text { and } \quad \sigma_{\delta}^{\alpha} f(x):=\frac{1}{\delta} \int_{0}^{\delta}\left|\Delta_{t}^{\alpha} f(x)\right| d t, \quad x \in \mathbb{T} .
$$

Using the boundedness of the Hardy-Littlewood Maximal operator in $L_{M, \omega}(\mathbb{T})$ for $M \in \bar{\Phi}_{p}, p>0$, and $\omega \in A_{p}$, we get

$$
\begin{gathered}
\left\|\sigma_{\delta}^{\alpha} f\right\|_{\langle M\rangle, \omega} \leq C(\alpha, M, \omega)\|f\|_{\langle M\rangle, \omega}<\infty \\
\left\|\mathcal{A}_{h} f\right\|_{\langle M\rangle, \omega} \leq C(M, \omega)\|f\|_{\langle M\rangle, \omega}<\infty .
\end{gathered}
$$


Using these facts and setting $x, h \in \mathbb{T}, r \geq 0$ we define via Binomial expansion that

$$
\begin{aligned}
\bar{\sigma}_{h}^{r} f(x) & :=\left(I-\mathcal{A}_{h}\right)^{r} f(x) \\
& =\sum_{k=0}^{\infty}(-1)^{k}\left(\begin{array}{c}
r \\
k
\end{array}\right) \frac{1}{h^{k}} \int_{-h / 2}^{h / 2} \ldots \int_{-h / 2}^{h / 2} f\left(x+u_{1}+\ldots+u_{k}\right) d u_{1} \ldots d u_{k},
\end{aligned}
$$

where $f \in L_{M, \omega}(\mathbb{T})$ and $x \in \mathbb{T}$. Then we have

$$
\left\|\bar{\sigma}_{h}^{r} f\right\|_{\langle M\rangle, \omega} \leq C(r, M, \omega)\|f\|_{\langle M\rangle, \omega}<\infty .
$$

Now, we define the following two type weighted fractional modulus of smoothness: if $r \in \mathbb{R}^{+} \cup\{0\}, M \in \bar{\Phi}_{p}, p>1$, and $\omega \in A_{p}$ we define for a function $f \in L_{M, \omega}(\mathbb{T})$

$$
\begin{aligned}
& \bar{\Omega}_{r}(f, h)_{M, \omega}:=\sup _{|\delta| \leq h}\left\|\sigma_{\delta}^{r} f\right\|_{\langle M\rangle, \omega}, \quad h \geq 0, \\
& \Omega_{r}(f, \delta)_{M, \omega}:=\sup _{0<h_{i}, t \leq \delta}\left\|\prod_{i=1}^{[r]}\left(I-\mathcal{A}_{h_{i}}\right) \bar{\sigma}_{t}^{r-[r]} f\right\|_{\langle M\rangle, \omega}, \delta \geq 0,
\end{aligned}
$$

where $\bar{\Omega}_{0}(f, \delta)_{M, \omega}=\Omega_{0}(f, \delta)_{M, \omega}=:\|f\|_{M, \omega} ; \quad \prod_{i=1}^{0}\left(I-\mathcal{A}_{h_{i}}\right) \bar{\sigma}_{t}^{r} f:=\bar{\sigma}_{t}^{r} f$ for $0<$ $r<1$, and $[r]$ denotes the integer part of the real number $r$.

In this case

$$
\bar{\Omega}_{r}(f, \delta)_{M, \omega} \leq C(r, M, \omega)\|f\|_{\langle M\rangle, \omega}
$$

and

$$
\Omega_{r}(f, \delta)_{M, \omega} \leq C(r, M, \omega)\|f\|_{\langle M\rangle, \omega} .
$$

$\sim$ The concept of fractional modulus of smoothness is not new (see for example ․ $[\mathrm{BDGS}]$ and $[\mathrm{Tab}])$.

We set $E_{n}(f)_{M, \omega}:=\inf \left\{\|f-T\|_{\langle M\rangle, \omega}: T \in \mathcal{T}_{n}\right\}$ for $f \in L_{M, \omega}(\mathbb{T})$, where $\mathcal{T}_{n}$ is the class of trigonometric polynomials of degree not greater than $n$. For a given $f \in L^{1}(\mathbb{T})$, let

$$
f(x) \backsim \frac{a_{0}(f)}{2}+\sum_{k=1}^{\infty}\left(a_{k}(f) \cos k x+b_{k}(f) \sin k x\right)=: \sum_{k=0}^{\infty} A_{k}(x, f)
$$

and

$$
\tilde{f}(x) \backsim \sum_{k=1}^{\infty}\left(a_{k}(f) \sin k x-b_{k}(f) \cos k x\right)=: \sum_{k=1}^{\infty} A_{k}(x, \tilde{f})
$$


be the Fourier and the conjugate Fourier series of $f$. We define the partial sum of Fourier series of $f$ as

$$
S_{n}(f):=S_{n}(x, f):=\sum_{k=0}^{n} A_{k}(x, f)
$$

for $n=0,1,2, \ldots$

Remark 2. (1) Using Extrapolation Theorem 1 and Remark 1 we get easily that if $M \in \bar{\Phi}_{p}, p>1, \omega \in A_{p}$, and $f \in L_{M, \omega}(\mathbb{T})$, then the operators $S_{n}: L_{M, \omega}(\mathbb{T}) \rightarrow$ $L_{M, \omega}(\mathbb{T})$ and $\tilde{f}: L_{M, \omega}(\mathbb{T}) \rightarrow L_{M, \omega}(\mathbb{T})$ are bounded in $L_{M, \omega}(\mathbb{T})$. Hence we get $\|\tilde{f}\|_{\langle M\rangle, \omega} \leq C\|f\|_{\langle M\rangle, \omega},\left\|S_{n}(f)\right\|_{\langle M\rangle, \omega} \leq C\|f\|_{\langle M\rangle, \omega}$ and $\left\|f-S_{n}(f)\right\|_{\langle M\rangle, \omega} \leq$ $C E_{n}(f)_{M, \omega}$ for $n=0,1,2, \ldots$ Furthermore

$$
E_{n}(f)_{M, \omega} \sim E_{n}(\tilde{f})_{M, \omega} .
$$

(2) If $M \in \bar{\Phi}_{p}, p>1$, and $\omega \in A_{p}$, then the hypotheses of Lemma 3 of [Kh] are fulfilled and therefore the set of trigonometric polynomials is a dense subset of $L_{M, \omega}(\mathbb{T})$. Then the approximation problems make sense in $L_{M, \omega}(\mathbb{T})$. Hence $E_{n}(f)_{M, \omega} \rightarrow 0$ as $n \rightarrow \infty$ and therefore the Fourier series of $f$ converges to $f$ in norm in $L_{M, \omega}(\mathbb{T})$, namely,

$$
f(x)=\sum_{k=1}^{\infty} A_{k}(x, f) .
$$

Let $\alpha \in \mathbb{R}^{+}$be given. We define fractional derivative (in the sense of Weyl) of a function $f \in L^{1}(\mathbb{T})$ as

$$
f^{(\alpha)}(x):=\frac{a_{0}(f)}{2}+\sum_{k=1}^{\infty} k^{\alpha} A_{k}\left(x+\frac{\alpha \pi}{2 k}, f\right)=: \sum_{k=0}^{\infty} A_{k}\left(x, f^{(\alpha)}\right) .
$$

Let $W_{M, \omega}^{\alpha}, \alpha>0$, be the class of functions $f \in L_{M, \omega}(\mathbb{T})$ such that $f^{(\alpha)} \in$ $\stackrel{N}{\sqsupset} L_{M, \omega}(\mathbb{T}) . W_{M, \omega}^{\alpha}$ becomes a Banach space with respect to the norm

$$
\|f\|_{W_{M, \omega}^{\alpha}}:=\|f\|_{\langle M\rangle, \omega}+\left\|f^{(\alpha)}\right\|_{\langle M\rangle, \omega} .
$$

The following two approximation theorems can be proved the same methods given in [A, Proposition 1] and [IG, Theorems 2 and 4]:

Theorem 2. If $M \in \bar{\Phi}_{p}, p>1, \omega \in A_{p}$, and $f \in L_{M, \omega}(\mathbb{T})$, then there is a positive constant $C$ depending only on $r, \omega$ and $M$ such that

$$
E_{n}(f)_{M, \omega} \leq C \Omega_{r}\left(f, \frac{1}{n+1}\right)_{M, \omega}
$$

holds for $r \in \mathbb{R}^{+}$and $n=0,1,2,3, \ldots$ 
Theorem 3. If $M \in \bar{\Phi}_{p}, p>1, \omega \in A_{p}$, and $f \in L_{M, \omega}(\mathbb{T})$, then there exists a positive constant $C$ depending only on $r, \omega$ and $M$ such that the inequality

$$
\Omega_{r}\left(f, \frac{1}{n}\right)_{M, \omega} \leq \frac{C}{n^{2 r}}\left\{E_{0}(f)_{M, \omega}+\sum_{\nu=1}^{n} \nu^{2 r-1} E_{\nu}(f)_{M, \omega}\right\}
$$

holds for $r \in \mathbb{R}^{+}$and $n=1,2,3, \ldots$

Using the methods of [AI, Theorems 1,3 and 4] we obtain

Theorem 4. Let $M \in \bar{\Phi}_{p}, p>1, \omega \in A_{p}$, and $f \in L_{M, \omega}(\mathbb{T})$. If

$$
\sum_{\nu=1}^{\infty} \nu^{\alpha-1} E_{\nu}(f)_{M, \omega}<\infty
$$

for some $\alpha \in(0, \infty)$, then $f \in W_{M, \omega}^{\alpha}$ and there is a positive constant $C$ depending only on $\alpha, \omega$ and $M$ such that

$$
E_{n}\left(f^{(\alpha)}\right)_{M, \omega} \leq C\left((n+1)^{\alpha} E_{n}(f)_{M, \omega}+\sum_{\nu=n+1}^{\infty} \nu^{\alpha-1} E_{\nu}(f)_{M, \omega}\right)
$$

holds for $r \in \mathbb{R}^{+}$and $n=0,1,2,3, \ldots$

Theorem 5. Let $M \in \bar{\Phi}_{p}, p>1, \omega \in A_{p}$, and $f \in L_{M, \omega}(\mathbb{T})$. If $r \in \mathbb{R}^{+}$and

$$
\sum_{\nu=1}^{\infty} \nu^{\alpha-1} E_{\nu}(f)_{M, \omega}<\infty
$$

for some $\alpha>0$, then there exists a positive constant $C$ depending only on $\alpha, \omega$, $r$ and $M$ such that

$$
\begin{aligned}
\Omega_{r}\left(f^{(\alpha)}, \frac{1}{n+1}\right)_{M, \omega} \leq & C\left(\frac{1}{(n+1)^{2 r}} \sum_{\nu=0}^{n}(\nu+1)^{\alpha+2 r-1} E_{\nu}(f)_{M, \omega}\right. \\
& \left.+\sum_{\nu=n+1}^{\infty} \nu^{\alpha-1} E_{\nu}(f)_{M, \omega}\right)
\end{aligned}
$$

holds for $n=0,1,2,3, \ldots$.

Theorem 6. If $M \in \bar{\Phi}_{p}, p>1, \omega \in A_{p}, f \in L_{M, \omega}(\mathbb{T})$ and $\alpha \in[0, \infty)$, then there exist a near best approximant $T \in \mathcal{T}_{n}$ for $f$ and a constant $C>0$ depending only on $\alpha, \omega$ and $M$ such that

$$
\left\|f^{(\alpha)}-T^{(\alpha)}\right\|_{\langle M\rangle, \omega} \leq C E_{n}\left(f^{(\alpha)}\right)_{M, \omega}
$$

holds. 
These theorems have plenty of applications in imbedding of function classes ([PS, ST]); in the study of absolutely convergent Fourier series ([S]), investigation of properties of the conjugate functions ([BS]), and characterizations of Lipschitz classes ([Kon, ST, TiM, TiM1]).

In the particular case $M(x, p)=x^{p}, 1<p<\infty, L^{p}(\mathbb{T}, \omega)=L_{M(\cdot, p), \omega}(\mathbb{T})$, the author proved in $[\mathrm{A}]$ that taking $\gamma_{1}:=\min \{2, p\}, \beta_{1}:=\max \{2, p\}$ direct and inverse inequalities are improved for $A_{p}$ weights:

Theorem 7. Let $1<p<\infty, \omega \in A_{p}$ and $f \in L^{p}(\mathbb{T}, \omega)$. If $n \in \mathbb{N}$ and $r \in \mathbb{R}^{+}$, then there exist positive constants $C$ and $C^{\prime}$ depending only on $r, \omega$ and $p$ such that

$$
\begin{aligned}
\frac{C}{n^{2 r}}\left\{\sum_{\nu=1}^{n} \nu^{2 \beta_{1} r-1} E_{\nu-1}^{\beta_{1}}(f)_{M(\cdot, p), \omega}\right\}^{1 / \beta_{1}} & \leq \Omega_{r}\left(f, \frac{1}{n}\right)_{p, \omega} \\
& \leq \frac{C^{\prime}}{n^{2 r}}\left\{\sum_{\nu=1}^{n} \nu^{2 \gamma_{1} r-1} E_{\nu-1}^{\gamma_{1}}(f)_{M(\cdot, p), \omega}\right\}^{1 / \gamma_{1}}
\end{aligned}
$$

holds.

This result motivates us to consider the refined/exact versions for Theorems 2 and 3. We will prove the refined/exact versions of Theorems 2 and 3. To prove refined inequalities we will need the following Marcinkiewicz Multiplier and Littlewood-Paley type theorems: Considering the results of Kurtz [Ku, Theorems 1 and 2 (periodic case)] we can obtain by Extrapolation Theorem 1 and Remark 1 that

Theorem 8. Let a sequence $\left\{\lambda_{\mu}\right\}$ of real numbers be satisfy

$$
\left|\lambda_{\mu}\right| \leq A, \quad \sum_{\mu=2^{m-1}}^{2^{m}-1}\left|\lambda_{\mu}-\lambda_{\mu+1}\right| \leq A
$$

for all $\mu, m \in \mathbb{N}$, where $A$ does not depend on $\mu$ and $m$. If $M \in \bar{\Phi}_{p}, p>1$, $\omega \in A_{p}$, and $f \in L_{M, \omega}(\mathbb{T})$, then there is a function $F \in L_{M, \omega}(\mathbb{T})$ such that the series $\sum_{k=0}^{\infty} \lambda_{k} A_{k}(\cdot, f)$ is Fourier series for $F$ and

$$
\|F\|_{\langle M\rangle, \omega} \leq C A\|f\|_{\langle M\rangle, \omega}
$$

holds with a positive constant $C$, non-depending on $f$.

Theorem 9. If $M \in \bar{\Phi}_{p}, p>1, \omega \in A_{p}$, and $f \in L_{M, \omega}(\mathbb{T})$, then

$$
\left\|\left(\sum_{\mu=\nu}^{\infty}\left|\Delta_{\mu}\right|^{2}\right)^{1 / 2}\right\|_{\langle M\rangle, \omega} \sim\left\|\sum_{\mu=2^{\nu-1}}^{\infty} A_{\nu}(x, f)\right\|_{\langle M\rangle, \omega}
$$


holds for some constants depending only on $M$ and $\omega$, where

$$
\Delta_{\mu}:=\Delta_{\mu}(\cdot, f):=\sum_{\nu=2^{\mu-1}}^{2^{\mu}-1} A_{\nu}(\cdot, f) .
$$

The following fractional analog of Bernstein inequality can be proved by the method given in [Ky3]:

Lemma 1. If $M \in \bar{\Phi}_{p}, p>1, \omega \in A_{p}, f \in L_{M, \omega}(\mathbb{T})$ and $n \in \mathbb{N}$, then there exists a positive constant $C$ depending only on $\alpha, \omega$ and $M$ such that the inequality

$$
\left\|T_{n}^{(\alpha)}\right\|_{M, \omega} \leq C n^{\alpha}\left\|T_{n}\right\|_{M, \omega}
$$

holds for $\alpha \in \mathbb{R}^{+}$.

1.1. Main results. Let $M \in \bar{\Phi}_{p}, 1<p \leq q, \omega \in A_{p}, f \in L_{M, \omega}(\mathbb{T})$ and we set $\beta:=\max (2, q-\delta)$ and $\gamma:=\min (2, p+\varepsilon)$ where $\varepsilon, \delta$ are some small positive numbers. The following theorem is an improvement of Theorem 2 :

Theorem 10. If $M \in \bar{\Phi}_{p}, p>1, \omega \in A_{p}, f \in L_{M, \omega}(\mathbb{T}), n \in \mathbb{N}$, and $r \in \mathbb{R}^{+}$, then there is a positive constant $C$ depending only on $r, \omega$ and $M$ such that

$$
\frac{C}{n^{2 r}}\left\{\sum_{\nu=1}^{n} \nu^{2 \beta r-1} E_{\nu}^{\beta}(f)_{M, \omega}\right\}^{1 / \beta} \leq \Omega_{r}\left(f, \frac{1}{n}\right)_{M, \omega}
$$

holds.

We have also an improvement of Theorem 3:

Theorem 11. Let $M \in \bar{\Phi}_{p}, p>1, \omega \in A_{p}, f \in L_{M, \omega}(\mathbb{T}), n \in \mathbb{N}, r \in \mathbb{R}^{+}$and $\Gamma(x):=M(\sqrt{x})$ be quasiconvex. Then there is a positive constant $C$ depending only on $r, \omega$ and $M$ such that

$$
\Omega_{r}\left(f, \frac{1}{n}\right)_{M, \omega} \leq \frac{C}{n^{2 r}}\left\{\sum_{\nu=1}^{n} \nu^{2 \gamma r-1} E_{\nu}^{\gamma}(f)_{M, \omega}\right\}^{1 / \gamma}
$$

I holds.

Theorem 11 is exact in the sense of order: Let $\left\{\alpha_{\mu}\right\}$ be a monotonic sequence of positive numbers converging to zero and we suppose $M \in \bar{\Phi}_{p}, p>1$ and $\omega \in A_{p}$. Let $\mathfrak{B}_{M, p}\left(\alpha_{\mu}\right)$ be the set of functions $f \in L_{M, \omega}(\mathbb{T})$ for which $E_{\mu}(f)_{M, \omega} \leq C \alpha_{\mu}$ for some constant $C$, non-depending on $f$.

Theorem 12. Let $2<p<q$. Then for every $\alpha_{\mu} \downarrow 0$, there exist $f_{1} \in \mathfrak{B}_{M, p}\left(\alpha_{\mu}\right)$ and a positive constant $C$ depending only on $M, \omega$ such that

$$
\frac{C}{n^{2}}\left\{\sum_{\nu=1}^{n} \nu^{3} \alpha_{\nu}^{2}\right\}^{1 / 2} \leq \Omega_{1}\left(f_{1}, \frac{1}{n}\right)_{M, \omega}
$$

holds for $n \in \mathbb{N}$. 
As a corollary of Theorems 10 and 11 we have the following version of Marchaud inequality and its inverse:

Corollary 1. Under the conditions of Theorems 10 and 11, if $r, l \in \mathbb{R}^{+}, r<l$ and $0<t \leq 1 / 2$, then there exist positive constants $C$ and $C^{\prime}$ depending only on $l, r, \omega$ and $M$ such that

$$
\begin{aligned}
C t^{2 r}\left\{\int_{t}^{1}\left[\frac{\Omega_{l}(f, u)_{M, \omega}}{u^{2 r}}\right]^{\beta} \frac{d u}{u}\right\}^{1 / \beta} & \leq \Omega_{r}(f, t)_{M, \omega} \\
& \leq C^{\prime 2 r}\left\{\int_{t}^{1}\left[\frac{\Omega_{l}(f, u)_{M, \omega}}{u^{2 r}}\right]^{\gamma} \frac{d u}{u}\right\}^{1 / \gamma}
\end{aligned}
$$

hold.

Improvements of Theorems 4 and 5 are

Theorem 13. Let $M \in \bar{\Phi}_{p}, p>1, \omega \in A_{p}$ and $f \in L_{M, \omega}(\mathbb{T})$. If

$$
\sum_{k=1}^{\infty} k^{\gamma \alpha-1} E_{k}^{\gamma}(f)_{M, \omega}<\infty
$$

for some $\alpha \in \mathbb{R}^{+}$, then $f \in W_{M, \omega}^{\alpha}$ and there exists a constant $C>0$ depending only on $\alpha, \omega$ and $M$ such that

$$
E_{n}\left(f^{(\alpha)}\right)_{M, \omega} \leq C\left(n^{\alpha} E_{n}(f)_{M, \omega}+\left\{\sum_{\nu=n+1}^{\infty} \nu^{\alpha \gamma-1} E_{\nu}^{\gamma}(f)_{M, \omega}\right\}^{1 / \gamma}\right)
$$

holds for $n \in \mathbb{N}$.

Corollary 2. Under the conditions of Theorem 13, there exists a constant $C>0$ I depending only on $\alpha, \omega, r$ and $M$ such that

$$
\begin{aligned}
\Omega_{r}\left(f^{(\alpha)}, \frac{1}{n}\right)_{M, \omega} \leq & C\left(\frac{1}{n^{2 r}}\left(\sum_{\nu=1}^{n} \nu^{\gamma(2 r+\alpha)-1} E_{\nu}^{\gamma}(f)_{M, \omega}\right)^{\frac{1}{\gamma}}\right. \\
& \left.+\left(\sum_{\nu=n+1}^{\infty} \nu^{\alpha \gamma-1} E_{\nu}^{\gamma}(f)_{M, \omega}\right)^{\frac{1}{\gamma}}\right)
\end{aligned}
$$

holds for $n \in \mathbb{N}$ and $\alpha, r \in \mathbb{R}^{+}$.

The following theorem improves, for even integers, the Jackson type direct theorem that obtained in [Ky2]: 
Theorem 14. Let $M \in \bar{\Phi}_{p}, p>1, \omega \in A_{p}, f \in L_{M, \omega}(\mathbb{T}), n \in \mathbb{N}$, and $r$ be an even integer. Then there is a positive constant $C$ depending only on $r, \omega$ and $M$ such that the inequality

$$
\frac{C}{n^{r}}\left\{\sum_{\nu=1}^{n} \nu^{\beta r-1} E_{\nu-1}^{\beta}(f)_{M, \omega}\right\}^{1 / \beta} \leq \bar{\Omega}_{r}\left(f, \frac{1}{n}\right)_{M, \omega}
$$

holds.

\section{Proofs of the Results}

Proof of Theorem 10. Let $r \in \mathbb{R}^{+}, \beta \in(1, \infty), n \in \mathbb{N}$ and we suppose that the number $m \in \mathbb{N}$ satisfies $2^{m} \leq n<2^{m+1}$. Using $E_{n}(f)_{M, \omega} \downarrow 0$ and LittlewoodPaley inequality (1.6) we have

$$
\begin{aligned}
J_{n, r}^{\beta} & :=\frac{1}{n^{2 r}}\left\{\sum_{\nu=1}^{n} \nu^{2 \beta r-1} E_{\nu}^{\beta}(f)_{M, \omega}\right\}^{\frac{1}{\beta}} \leq \frac{1}{n^{2 r}}\left\{\sum_{\nu=1}^{m+1} \sum_{\mu=2^{\nu-1}}^{2^{\nu}-1} \mu^{2 \beta r-1} E_{\mu}^{\beta}(f)_{M, \omega}\right\}^{\frac{1}{\beta}} \\
& \leq \frac{1}{n^{2 r}}\left\{\sum_{\nu=1}^{m+1} 2^{2 \nu \beta r} E_{2^{\nu-1}-1}^{\beta}(f)_{M, \omega}\right\} \\
& \leq \frac{C}{n^{2 r}}\left\{\sum_{\nu=1}^{m+1} 2^{2 \nu \beta r}\left\|\sum_{\mu=2^{\nu-1}}^{\infty} A_{\mu}(x, f)\right\|_{\langle M\rangle, \omega}^{\beta}\right\}^{1 / \beta} \\
& \leq \frac{C}{n^{2 r}}\left\{\sum_{\nu=1}^{m+1} 2^{2 \nu \beta r} \|\left(\sum_{\mu=\nu}^{\infty}\left|\Delta_{\mu}\right|^{2} \|_{\langle M\rangle, \omega}^{1 / \beta}\right\}^{1 / \beta}\right. \\
& =C\left\{\sum_{\nu=1}^{m+1} \|\left(\frac{2^{4 \nu r}}{n^{4 r}} \sum_{\mu=\nu}^{\infty}\left|\Delta_{\mu}\right|^{2}\left\|_{\langle M\rangle, \omega}^{1 / 2}\right\|^{\beta} .\right.\right.
\end{aligned}
$$

We assume $\beta=2$. Then $2 \geq(q-\delta)$ and

$$
J_{n, r}^{2} \leq C\left\{\sum_{\nu=1}^{m+1}\left\|\left(\frac{2^{4 \nu r}}{n^{4 r}} \sum_{\mu=\nu}^{\infty}\left|\Delta_{\mu}\right|^{2}\right)^{1 / 2}\right\|_{\langle M\rangle, \omega}^{2}\right\}^{1 / 2} .
$$

Since the norm of $l_{p}$ is decrease with $p \uparrow$ we have

$$
J_{n, r}^{2} \leq C\left\{\sum_{\nu=1}^{m+1}\left\|\left(\frac{2^{4 \nu r}}{n^{4 r}} \sum_{\mu=\nu}^{\infty}\left|\Delta_{\mu}\right|^{2}\right)^{1 / 2}\right\|_{\langle M\rangle, \omega}^{q-\delta}\right\}^{1 /(q-\delta)} .
$$


Using $(q-\delta)$ concavity of $L_{M, \omega}(\mathbb{T})$ we obtain

$$
\begin{aligned}
J_{n, r}^{2} & \leq C\left\|\left(\sum_{\nu=1}^{m+1}\left(\frac{2^{4 \nu r}}{n^{4 r}} \sum_{\mu=\nu}^{\infty}\left|\Delta_{\mu}\right|^{2}\right)^{(q-\delta) / 2}\right)^{1 /(q-\delta)}\right\|_{\langle M\rangle, \omega} \\
& \leq C\left\|\sum_{\nu=1}^{m+1} \frac{2^{2 \nu r}}{n^{2 r}} \sum_{\mu=\nu}^{\infty}\left|\Delta_{\mu}\right|\right\|_{\langle M\rangle, \omega} .
\end{aligned}
$$

Abel's transformation and Minkowski's inequality imply that

$$
\begin{aligned}
J_{n, r}^{2} & \leq C\left\|\sum_{\nu=1}^{m} \frac{2^{2 \nu r}}{n^{2 r}}\left|\Delta_{\nu}\right|+\frac{2^{2 r(m+1)}}{n^{2 r}} \sum_{\mu=m+1}^{\infty}\left|\Delta_{\mu}\right|\right\|_{\langle M\rangle, \omega} \\
& \leq C\left\|\sum_{\nu=1}^{m} \frac{2^{2 \nu r}}{n^{2 r}}\left|\Delta_{\nu}\right|\right\|_{\langle M\rangle, \omega}+C\left\|\frac{2^{2 r(m+1)}}{n^{2 r}} \sum_{\mu=m+1}^{\infty}\left|\Delta_{\mu}\right|\right\|_{\langle M\rangle, \omega} .
\end{aligned}
$$

If $\beta=q-\delta$, then $2 \leq(q-\delta)$ and $(q-\delta)$ concavity of $L_{M, \omega}(\mathbb{T})$ imply that

$$
\begin{aligned}
J_{n, r}^{q-\delta} & \leq C\left(\sum_{\nu=1}^{m+1}\left\|\left(\frac{2^{4 \nu r}}{n^{4 r}} \sum_{\mu=\nu}^{\infty}\left|\Delta_{\mu}\right|^{2}\right)^{1 / 2}\right\|_{\langle M\rangle, \omega}^{q-\delta}\right)^{\frac{1}{q-\delta}} \\
& \leq C\left(\left\|\left(\sum_{\nu=1}^{m+1}\left(\frac{2^{4 \nu r}}{n^{4 r}} \sum_{\mu=\nu}^{\infty}\left|\Delta_{\mu}\right|^{2}\right)^{\frac{q-\delta}{2}}\right)^{\frac{1}{q-\delta}}\right\|_{\langle M\rangle, \omega}\right) \\
& \leq C\left\|\left(\sum_{\nu=1}^{m+1} \frac{2^{4 \nu r}}{n^{4 r}} \sum_{\mu=\nu}^{\infty}\left|\Delta_{\mu}\right|^{2}\right)^{1 / 2}\right\|_{\langle M\rangle, \omega} .
\end{aligned}
$$

Using Abel's transformation and Minkowski's inequality we get

$$
\begin{aligned}
J_{n, r}^{q-\delta} & \leq C\left\|\left(\sum_{\nu=1}^{m} \frac{2^{4 \nu r}}{n^{4 r}}\left|\Delta_{\nu}\right|^{2}+\frac{2^{4 r(m+1)}}{n^{4 r}} \sum_{\mu=m+1}^{\infty}\left|\Delta_{\mu}\right|^{2}\right)^{1 / 2}\right\|_{\langle M\rangle, \omega} \\
& \leq C\left\|\left(\sum_{\nu=1}^{m} \frac{2^{4 \nu r}}{n^{4 r}}\left|\Delta_{\nu}\right|^{2}\right)^{\frac{1}{2}}\right\|_{\langle M\rangle, \omega}+C\left\|\left(\frac{2^{4 r(m+1)}}{n^{4 r}} \sum_{\mu=m+1}^{\infty}\left|\Delta_{\mu}\right|^{2}\right)^{\frac{1}{2}}\right\|_{\langle M\rangle, \omega} \\
& \leq C\left\|\sum_{\nu=1}^{m} \frac{2^{2 \nu r}}{n^{2 r}}\left|\Delta_{\nu}\right|\right\|_{\langle M\rangle, \omega}+C\left\|\sum_{\mu=m+1}^{\infty}\left|\Delta_{\mu}\right|\right\|_{\langle M\rangle, \omega} .
\end{aligned}
$$


Therefore we obtain

$$
J_{n, r}^{\beta} \leq C\left\|\sum_{\nu=1}^{m} \frac{2^{2 \nu r}}{n^{2 r}}\left|\Delta_{\nu}\right|\right\|_{\langle M\rangle, \omega}+C\left\|\sum_{\mu=m+1}^{\infty}\left|\Delta_{\mu}\right|\right\|_{\langle M\rangle, \omega} .
$$

Then

$$
J_{n, r}^{\beta} \leq C\left\|\sum_{\nu=1}^{m} \sum_{\mu=2^{\nu-1}}^{2^{\nu}-1} \frac{2^{2 \nu r}}{n^{2 r}} A_{\mu}(x, f)\right\|_{\langle M\rangle, \omega}+C\left\|\sum_{\mu=2^{m}}^{\infty} A_{\mu}(x, f)\right\|_{\langle M\rangle, \omega} .
$$

Since

we have

$$
\left\|f-S_{n}(f)\right\|_{\langle M\rangle, \omega} \leq C E_{n}(f)_{M, \omega}
$$

$$
\begin{aligned}
J_{n, r}^{\beta} \leq & C\left\|\sum_{\nu=1}^{m} \sum_{\mu=2^{\nu-1}}^{2^{\nu}-1} \frac{2^{2 \nu r}}{|\mu|^{2 r}} \frac{\left(\frac{|\mu|}{n}\right)^{2 r}}{\left(1-\frac{\sin \frac{\mu}{n}}{\frac{\mu}{n}}\right)^{r}}\left(1-\frac{\sin \frac{\mu}{n}}{\frac{\mu}{n}}\right)^{r} A_{\mu}(\cdot, f)\right\|_{\langle M\rangle, \omega} \\
& +C E_{2^{m}-1}(f)_{M, \omega}
\end{aligned}
$$

and using Theorem 2

$$
J_{n, r}^{\beta} \leq C\left\|\sum_{\mu=1}^{2^{m}-1} \frac{2^{2 \nu r}}{\mu^{2 r}} \frac{\left(\frac{\mu}{n}\right)^{2 r}}{\left(1-\frac{\sin \frac{\mu}{n}}{\frac{\mu}{n}}\right)^{r}}\left(1-\frac{\sin \frac{\mu}{n}}{\frac{\mu}{n}}\right)^{r} A_{\mu}(\cdot, f)\right\|_{\langle M\rangle, \omega}+C \Omega_{r}\left(f, \frac{1}{n}\right)_{M, \omega} .
$$

Now we define

$$
h_{\mu}:= \begin{cases}\frac{2^{2 \nu r}}{\mu^{2 r}} & , \text { for } 1 \leq \mu \leq 2^{m}-1, \nu=1, \ldots, m \\ \frac{2^{2 m r}}{\mu^{2 r}} & , \text { for } 2^{m} \leq \mu \leq n \\ 0 & , \text { for } \mu>n\end{cases}
$$

and

$$
\lambda_{\mu}:= \begin{cases}\frac{\left(\frac{\mu}{n}\right)^{2 r}}{\left(1-\frac{\sin \frac{\mu}{n}}{\frac{\mu}{n}}\right)^{r}} & , \text { for } 1 \leq \mu \leq n, \\ 0 & , \text { for } \mu>n .\end{cases}
$$

Hence, for $\mu=1,2,3, \ldots,\left\{h_{\mu}\right\}$ satisfy (1.5) with $A=2^{2 r}$ and $\left\{\lambda_{\mu}\right\}$ satisfy (1.5) with $A=(1-\sin 1)^{-r}$. Therefore taking

we get

$$
I:=\left\|\sum_{\mu=1}^{2^{m}-1} \frac{2^{2 \nu r}}{\mu^{2 r}} \frac{\left(\frac{\mu}{n}\right)^{2 r}}{\left(1-\frac{\sin \frac{\mu}{n}}{\frac{\mu}{n}}\right)^{r}}\left(1-\frac{\sin \frac{\mu}{n}}{\frac{\mu}{n}}\right)^{r} A_{\mu}(\cdot, f)\right\|_{\langle M\rangle, \omega}
$$

$$
I=\left\|\sum_{\mu=1}^{\infty} h_{\mu} \lambda_{\mu}\left(1-\frac{\sin \frac{\mu}{n}}{\frac{\mu}{n}}\right)^{r} A_{\mu}(\cdot, f)\right\|_{\langle M\rangle, \omega}
$$


and using Theorem 8 twice there hold

$$
\begin{aligned}
J_{n, r}^{\beta} & \leq \frac{C 2^{2 r}}{(1-\sin 1)^{r}}\left\|\sum_{\mu=1}^{\infty}\left(1-\frac{\sin \frac{\mu}{n}}{\frac{\mu}{n}}\right)^{r} A_{\mu}(x, f)\right\|_{\langle M\rangle, \omega} \\
& \leq \frac{C 2^{2 r}}{(1-\sin 1)^{r}}\left\|\left(I-\sigma_{1 / n}\right)^{r} f\right\|_{\langle M\rangle, \omega} \\
& =\frac{C 2^{2 r}}{(1-\sin 1)^{r}}\left\|\left(I-\sigma_{1 / n}\right)^{[r]}\left(I-\sigma_{1 / n}\right)^{r-[r]} f\right\|_{\langle M\rangle, \omega} \\
& \leq \frac{C 2^{2 r}}{(1-\sin 1)^{r}} \sup _{0<h_{i}, t<\frac{1}{n}}\left\|\prod_{i=1}^{[r]}\left(I-\sigma_{h_{i}}\right)\left(I-\sigma_{t}\right)^{r-[r]} f\right\|_{\langle M\rangle, \omega} \\
& \leq C \Omega_{r}\left(f, \frac{1}{n}\right)_{M, \omega} .
\end{aligned}
$$

Therefore

$$
J_{n, r}^{\beta} \leq C \Omega_{r}\left(f, \frac{1}{n}\right)_{M, \omega}
$$

Proof of Theorem 10 is completed.

Proof of Theorem 11. As it is well-known that the expression $\sigma_{t, h_{1}, h_{2}, \ldots, h_{[r]}}^{r} f:=$ $\prod_{i=1}^{[r]}\left(I-\sigma_{h_{i}}\right)\left(I-\sigma_{t}\right)^{r-[r]} f$ has Fourier series

$\sigma_{t, h_{1}, h_{2}, \ldots, h_{[r]}}^{r} f \sim \sum_{\nu=1}^{\infty}\left(1-\frac{\sin \nu t}{\nu t}\right)^{r-[r]}\left(1-\frac{\sin \nu h_{1}}{\nu h_{1}}\right) \ldots\left(1-\frac{\sin \nu h_{[r]}}{\nu h_{[r]}}\right) A_{\nu}(\cdot, f)$

and

$$
\sigma_{t, h_{1}, h_{2}, \ldots, h_{[r]}}^{r} f(\cdot)=\sigma_{t, h_{1}, h_{2}, \ldots, h_{[r]}}^{r}\left(f(\cdot)-S_{2^{m-1}}(\cdot, f)\right)+\sigma_{t, h_{1}, h_{2}, \ldots, h_{[r]}}^{r} S_{2^{m-1}}(\cdot, f) .
$$

$\stackrel{N}{i}$ From $E_{n}(f)_{M, \omega} \downarrow 0$ we have

$$
\begin{aligned}
\left\|\sigma_{t, h_{1}, h_{2}, \ldots, h_{[r]}}^{r}\left(f(\cdot)-S_{2^{m-1}}(\cdot, f)\right)\right\|_{\langle M\rangle, \omega} & \leq C\left\|f-S_{2^{m-1}}(f)\right\|_{\langle M\rangle, \omega} \\
& \leq C E_{2^{m-1}}(f)_{M, \omega} \\
& \leq \frac{C}{n^{2 r}}\left\{\sum_{\nu=1}^{n} \nu^{2 \gamma r-1} E_{\nu}^{\gamma}(f)_{M, \omega}\right\}^{1 / \gamma} .
\end{aligned}
$$

On the other hand from (1.6) we get

$$
\left\|\sigma_{t, h_{1}, h_{2}, \ldots, h_{[r]}}^{r} S_{2^{m-1}}(f)\right\|_{\langle M\rangle, \omega} \leq C\left\|\left\{\sum_{\mu=1}^{m}\left|\delta_{\mu}\right|^{2}\right\}^{1 / 2}\right\|_{\langle M\rangle, \omega}
$$


where

$$
\delta_{\mu}:=\sum_{\nu=2^{\mu-1}}^{2^{\mu}-1}\left(1-\frac{\sin \nu t}{\nu t}\right)^{r-[r]}\left(1-\frac{\sin \nu h_{1}}{\nu h_{1}}\right) \ldots\left(1-\frac{\sin \nu h_{[r]}}{\nu h_{[r]}}\right) A_{\nu}(\cdot, f) .
$$

If $p+\varepsilon<2$, then $\gamma=p+\varepsilon$ and $(p+\varepsilon)$ convexity of $L_{M, \omega}(\mathbb{T})$ imply that

$$
\begin{aligned}
\left\|\left\{\sum_{\mu=1}^{m}\left|\delta_{\mu}\right|^{2}\right\}^{1 / 2}\right\|_{\langle M\rangle, \omega} & \leq C\left\|\left\{\sum_{\mu=1}^{m}\left|\delta_{\mu}\right|^{p+\varepsilon}\right\}^{1 /(p+\varepsilon)}\right\|_{\langle M\rangle, \omega} \\
& \leq C\left\{\sum_{\mu=1}^{m}\left\|\delta_{\mu}\right\|_{\langle M\rangle, \omega}^{p+\varepsilon}\right\}^{1 /(p+\varepsilon)} .
\end{aligned}
$$

If $p+\varepsilon>2$, then $\gamma=2$. Using $M \sim \Phi_{1}$ and quasiconvexity of $M(\sqrt{x})$ we get

$$
\begin{aligned}
& \left\|\left\{\sum_{\mu=1}^{m}\left|\delta_{\mu}\right|^{2}\right\}^{1 / 2}\right\|_{\langle M\rangle, \omega}=\inf \left\{\tau>0: \int_{\mathbb{T}} \Phi_{1}\left(\frac{\left\{\sum_{\mu=1}^{m}\left|\delta_{\mu}\right|^{2}\right\}^{1 / 2}}{\tau}\right) \omega(x) d x \leq 1\right\} \\
& \sim \inf \left\{\tau>0: \int_{\mathbb{T}} M\left(\frac{\left\{\sum_{\mu=1}^{m}\left|\delta_{\mu}\right|^{2}\right\}^{1 / 2}}{\tau}\right) \omega(x) d x \leq 1\right\} \\
& =\inf \left\{\tau>0: \int_{\mathbb{T}} \Gamma\left(\frac{\sum_{\mu=1}^{m}\left|\delta_{\mu}\right|^{2}}{\tau^{2}}\right) \omega(x) d x \leq 1\right\} \\
& =\left(\inf \left\{u>0: \int_{\mathbb{T}} \Gamma\left(\frac{\sum_{\mu=1}^{m}\left|\delta_{\mu}\right|^{2}}{u}\right) \omega(x) d x \leq 1\right\}\right)^{1 / 2} \\
& =\left\|\sum_{\mu=1}^{m}\left|\delta_{\mu}\right|^{2}\right\|_{\Gamma, \omega}^{1 / 2} \leq\left(\sum_{\mu=1}^{m}\left\|\left|\delta_{\mu}\right|^{2}\right\|_{\Gamma, \omega}\right)^{1 / 2} \\
& =\left(\sum_{\mu=1}^{m}\left\|\delta_{\mu}\right\|_{M, \omega}^{2}\right)^{1 / 2}
\end{aligned}
$$




$$
\begin{aligned}
& \sim\left(\sum_{\mu=1}^{m}\left(\inf \left\{\tau>0: \int_{\mathbb{T}} \Phi_{1}\left(\frac{\left|\delta_{\mu}\right|}{\tau}\right) \omega(x) d x \leq 1\right\}\right)^{2}\right)^{1 / 2} \\
& =\left(\sum_{\mu=1}^{m}\left\|\delta_{\mu}\right\|_{\langle M\rangle, \omega}^{2}\right)^{1 / 2} .
\end{aligned}
$$

Therefore we have

$$
\left\|\left\{\sum_{\mu=1}^{m}\left|\delta_{\mu}\right|^{2}\right\}^{1 / 2}\right\|_{\langle M\rangle, \omega} \leq C\left\{\sum_{\mu=1}^{m}\left\|\delta_{\mu}\right\|_{\langle M\rangle, \omega}^{\gamma}\right\}^{1 / \gamma} .
$$

We estimate $\left\|\delta_{\mu}\right\|_{\langle M\rangle, \omega}$. Since

$$
\begin{aligned}
\left\|\delta_{\mu}\right\|_{\langle M\rangle, \omega} & =\| \sum_{\nu=2^{\mu-1}}^{2^{\mu}-1}\left[\nu^{r}\left(1-\frac{\sin \nu t}{\nu t}\right)^{r-[r]}\left(1-\frac{\sin \nu h_{1}}{\nu h_{1}}\right) \ldots\left(1-\frac{\sin \nu h_{[r]}}{\nu h_{[r]}}\right)\right] \\
& \times\left[\frac{1}{\nu^{r}} A_{\nu}(x, f)\right] \|_{\langle M\rangle, \omega},
\end{aligned}
$$

using Abel's transformation we get

$$
\begin{aligned}
\left\|\delta_{\mu}\right\|_{\langle M\rangle, \omega} \leq & \sum_{\nu=2^{\mu-1}}^{2^{\mu}-2} \mid \nu^{r}\left(1 \frac{\sin \nu t}{\nu t}\right)^{r-[r]}\left(1-\frac{\sin \nu h_{1}}{\nu h_{1}}\right) \ldots\left(1-\frac{\sin \nu h_{[r]}}{\nu h_{[r]}}\right)- \\
& -(\nu+1)^{r}\left(1-\frac{\sin (\nu+1) t}{(\nu+1) t}\right)^{r-[r]}\left(1-\frac{\sin (\nu+1) h_{1}}{(\nu+1) h_{1}}\right) \ldots \\
& \ldots\left(1-\frac{\sin (\nu+1) h_{[r]}}{(\nu+1) h_{[r]}}\right) \mid\left\|\sum_{l=2^{\mu-1}}^{\nu} \frac{1}{l^{r}} A_{l}(x, f)\right\|_{\langle M\rangle, \omega} \\
+ & {\left[\left(2^{\mu}-1\right)^{r}\left(1-\frac{\sin \left(2^{\mu}-1\right) t}{\left(2^{\mu}-1\right) t}\right)^{r-[r]}\left(1-\frac{\sin \left(2^{\mu}-1\right) h_{1}}{\left(2^{\mu}-1\right) h_{1}}\right) \ldots\right.} \\
& \ldots\left|\left(1-\frac{\sin \left(2^{\mu}-1\right) h_{[r]}}{\left(2^{\mu}-1\right) h_{[r]}}\right)\right|\left\|\sum_{l=2^{\mu-1}}^{2^{\mu}-1} \frac{1}{l^{r}} A_{l}(x, f)\right\|_{\langle M\rangle, \omega} .
\end{aligned}
$$

Using Marcinkiewicz Multiplier Theorem we have

$$
\begin{aligned}
\left\|\sum_{l=2^{\mu-1}}^{2^{\mu}-1} \frac{1}{l^{r}} A_{l}(x, f)\right\|_{\langle M\rangle, \omega} & \leq C\left\|\sum_{l=2^{\mu-1}}^{2^{\mu}-1} A_{l}(x, f)\right\|_{\langle M\rangle, \omega} \\
& \leq C E_{2^{\mu-1}-1}(f)_{M, \omega}
\end{aligned}
$$


and similarly

$$
\left\|\sum_{l=2^{\mu-1}}^{\nu} \frac{1}{l^{r}} A_{l}(x, f)\right\|_{\langle M\rangle, \omega} \leq C E_{2^{\mu-1}-1}(f)_{M, \omega} .
$$

Since $x^{r}\left(1-\frac{\sin x}{x}\right)^{r}$ is non decreasing and $\left(1-\frac{\sin x}{x}\right) \leq x^{2}$ for $x>0$ we obtain

$$
\begin{aligned}
\left\|\delta_{\mu}\right\|_{\langle M\rangle, \omega} \leq & \frac{C}{t^{r-[r]} h_{1} \ldots h_{[r]}}\left[\sum_{\nu=2^{\mu-1}}^{2^{\mu}-2} \mid(\nu t)^{r-[r]}\left(1-\frac{\sin \nu t}{\nu t}\right)^{r-[r]}\left(\nu h_{1}\right) \times\right. \\
& \times\left(1-\frac{\sin \nu h_{1}}{\nu h_{1}}\right) \ldots\left(\nu h_{[r]}\right)\left(1-\frac{\sin \nu h_{[r]}}{\nu h_{[r]}}\right)-((\nu+1) t)^{r-[r]} \\
& \times\left(1-\frac{\sin (\nu+1) t}{(\nu+1) t}\right)^{r-[r]}\left((\nu+1) h_{1}\right)\left(1-\frac{\sin (\nu+1) h_{1}}{(\nu+1) h_{1}}\right) \ldots \\
& \left.\ldots\left((\nu+1) h_{[r]}\right)\left(1-\frac{\sin (\nu+1) h_{[r]}}{(\nu+1) h_{[r]}}\right) \mid\right] E_{2^{\mu-1}-1}(f)_{M, \omega} \\
& +\mid\left(\left(2^{\mu}-1\right) t\right)^{r-[r]}\left(1-\frac{\sin \left(2^{\mu}-1\right) t}{\left(2^{\mu}-1\right) t}\right)^{r-[r]}\left(2^{\mu}-1\right) \\
\times & h_{1}\left(1-\frac{\sin \left(2^{\mu}-1\right) h_{1}}{\left(2^{\mu}-1\right) h_{1}}\right) \ldots\left(2^{\mu}-1\right) h_{[r]}\left(1-\frac{\sin \left(2^{\mu}-1\right) h_{[r]}}{\left(2^{\mu}-1\right) h_{[r]}}\right) \mid \\
& \times E_{2^{\mu-1}-1}(f)_{M, \omega} \\
\leq & C\left(1-\frac{\sin \left(2^{\mu}-1\right) t}{\left(2^{\mu}-1\right) t}\right)^{r-[r]}\left(1-\frac{\sin \left(2^{\mu}-1\right) h_{1}}{\left(2^{\mu}-1\right) h_{1}}\right) \ldots \\
& \ldots\left(1-\frac{\sin \left(2^{\mu}-1\right) h_{[r]}}{\left(2^{\mu}-1\right) h_{[r]}}\right) \times E_{2^{\mu-1}-1}(f)_{M, \omega} \\
\leq & C 2^{2 \mu r} t^{2 r-2[r]} h_{1}^{2} \ldots h_{[r]}^{2} E_{2^{\mu-1}-1}(f)_{M, \omega}
\end{aligned}
$$

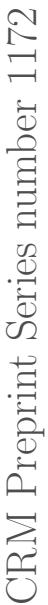

and therefore

$$
\left\|\delta_{\mu}\right\|_{\langle M\rangle, \omega} \leq C 2^{2 \mu r} t^{2(r-[r])} h_{1}^{2} \ldots h_{[r]}^{2} E_{2^{\mu-1}-1}(f)_{M, \omega} .
$$

Then

$$
\begin{aligned}
\left\|\sigma_{t, h_{1}, h_{2}, \ldots, h_{[r]}}^{r} S_{2^{m-1}}(f)\right\|_{\langle M\rangle, \omega} & \leq C t^{2(r-[r])} h_{1}^{2} \ldots h_{[r]}^{2}\left\{\sum_{\mu=1}^{m} 2^{2 \mu r \gamma} E_{2^{\mu-1}-1}^{\gamma}(f)_{M, \omega}\right\}^{\frac{1}{\gamma}} \\
& \leq C t^{2(r-[r])} h_{1}^{2} \ldots h_{[r]}^{2}\left\{2^{2 \gamma r} E_{0}^{\gamma}(f)_{M, \omega}\right\}^{\frac{1}{\gamma}}
\end{aligned}
$$




$$
\begin{aligned}
& +C t^{2(r-[r])} h_{1}^{2} \ldots h_{[r]}^{2}\left\{\sum_{\mu=2}^{m} \sum_{\nu=2^{\mu-2}+1}^{2^{\mu-1}} \nu^{2 \gamma r-1} E_{\nu}^{\gamma}(f)_{M, \omega}\right\}^{\frac{1}{\gamma}} \\
\leq & C t^{2(r-[r])} h_{1}^{2} \ldots h_{[r]}^{2}\left\{\sum_{\nu=1}^{2^{m-1}} \nu^{2 \gamma r-1} E_{\nu-1}^{\gamma}(f)_{M, \omega}\right\}^{\frac{1}{\gamma}} .
\end{aligned}
$$

The last inequality implies that

$$
\Omega_{r}\left(f, \frac{1}{n}\right)_{M, \omega} \leq \frac{C}{n^{2 r}}\left\{\sum_{\nu=1}^{n} \nu^{2 \gamma r-1} E_{\nu-1}^{\gamma}(f)_{M, \omega}\right\}^{1 / \gamma} .
$$

Theorem 11 is proved.

Proof of Theorem 12. Let $f_{1}(x)=\sum_{\nu=0}^{\infty} \sqrt{\left(\frac{\alpha_{2} \nu}{2^{\nu}}\right)^{2}-\left(\frac{\alpha_{2} \nu+1}{2^{\nu+1}}\right)^{2}} \sin 2^{\nu} x, x \in \mathbb{T}$. Then by $\left\|f_{1}\right\|_{\langle M\rangle, \omega} \leq C \frac{\alpha_{2}}{2}\|1\|_{\langle M\rangle, \omega}<\infty$ we have $f_{1} \in L_{M, \omega}(\mathbb{T})$. Moreover

$$
E_{\mu}\left(f_{1}\right)_{M, \omega} \leq C\left\|f_{1}-S_{\mu}\left(f_{1}\right)\right\|_{\langle M\rangle, \omega} \leq C \frac{\alpha_{2^{\mu}}}{2^{\mu}}\|1\|_{\langle M\rangle, \omega} \leq C \alpha_{\mu}
$$

and therefore $f_{1} \in \mathfrak{B}_{M, p}\left(\alpha_{\mu}\right)$. On the other hand

$$
\left(I-\mathcal{A}_{h}\right) f_{1}(x)=\sum_{\nu=0}^{\infty} \sqrt{\left(\frac{\alpha_{2^{\nu}}}{2^{\nu}}\right)^{2}-\left(\frac{\alpha_{2^{\nu+1}}}{2^{\nu+1}}\right)^{2}}\left(1-\frac{\sin 2^{\nu} h}{2^{\nu} h}\right) \sin 2^{\nu} x,
$$

and $\left\|\left(I-\mathcal{A}_{h}\right) f_{1}\right\|_{\langle M\rangle, \omega} \geq C\left\|\left(I-\mathcal{A}_{h}\right) f_{1}\right\|_{2, \omega}$. Now, there is a real number $a>1$ such that $\left\|\left(I-\mathcal{A}_{h}\right) f_{1}\right\|_{2, \omega} \geq C\left\|\left(I-\mathcal{A}_{h}\right) f_{1}\right\|_{a}$ (see for example Lemma 1 of $[\mathrm{Ky} 1])$. Since $f_{1}$ has lacunary Fourier series and

$$
\sum_{\nu=0}^{\infty}\left(\left(\frac{\alpha_{2^{\nu}}}{2^{\nu}}\right)^{2}-\left(\frac{\alpha_{2^{\nu+1}}}{2^{\nu+1}}\right)^{2}\right)\left(1-\frac{\sin 2^{\nu} h}{2^{\nu} h}\right)^{2}<\infty
$$

using Theorem 8.20 of [ZYG] we get

$$
\left\|\left(I-\mathcal{A}_{h}\right) f_{1}\right\|_{\langle M\rangle, \omega}^{2} \geq C \sum_{\nu=0}^{\infty}\left(\left(\frac{\alpha_{2^{\nu}}}{2^{\nu}}\right)^{2}-\left(\frac{\alpha_{2^{\nu+1}}}{2^{\nu+1}}\right)^{2}\right)\left(1-\frac{\sin 2^{\nu} h}{2^{\nu} h}\right)^{2} .
$$

We set $h=1 / n$. Since

$$
\sin ^{2} \frac{x}{2} \leq \frac{3}{2}\left(1-\frac{\sin x}{x}\right) \text { for every } x \in \mathbb{R}^{+}
$$

and

$$
\frac{2}{\pi} x \leq \sin x \text { for every } x \in[0, \pi / 2]
$$


we get

$$
\begin{aligned}
\left\|\left(I-\mathcal{A}_{1 / n}\right) f_{1}\right\|_{\langle M\rangle, \omega}^{2} & \geq C \sum_{\nu=0}^{n}\left(\left(\frac{\alpha_{2^{\nu}}}{2^{\nu}}\right)^{2}-\left(\frac{\alpha_{2^{\nu+1}}}{2^{\nu+1}}\right)^{2}\right)\left(1-\frac{\sin 2^{\nu} / n}{2^{\nu} / n}\right)^{2} \\
& \geq C \sum_{\nu=0}^{n}\left(\left(\frac{\alpha_{2^{\nu}}}{2^{\nu}}\right)^{2}-\left(\frac{\alpha_{2^{\nu+1}}}{2^{\nu+1}}\right)^{2}\right) \frac{\left(2^{\nu}\right)^{4}}{n^{4}} \\
& =\frac{C}{n^{4}} \sum_{\nu=0}^{n}\left(2^{\nu}\right)^{4}\left(\left(\frac{\alpha_{2^{\nu}}}{2^{\nu}}\right)^{2}-\left(\frac{\alpha_{2^{\nu+1}}}{2^{\nu+1}}\right)^{2}\right) \\
& \geq \frac{C}{n^{4}}\left(\sum_{\nu=1}^{n} \nu^{3} \alpha_{\nu}^{2}-n^{4} \alpha_{n+1}^{2}\right)
\end{aligned}
$$

Now we get

$$
\frac{C}{n^{4}}\left(\sum_{\nu=1}^{n} \nu^{3} \alpha_{\nu}^{2}\right) \leq \alpha_{n+1}^{2}+\left(\Omega_{1}\left(f, \frac{1}{n}\right)_{M, \omega}\right)^{2}
$$

Since

$$
\begin{aligned}
E_{n}\left(f_{1}\right)_{M, \omega}^{2} & \geq C\left\|f_{1}-S_{n}\left(f_{1}\right)\right\|_{\langle M\rangle, \omega}^{2} \geq C\left\|f_{1}-S_{n}\left(f_{1}\right)\right\|_{a}^{2} \\
& =C \sum_{k=n}^{\infty}\left(\alpha_{k}^{2}-\alpha_{k+1}^{2}\right)=C \alpha_{n}^{2}
\end{aligned}
$$

we get

$$
\begin{gathered}
\alpha_{n}^{2} \leq C E_{n}\left(f_{1}\right)_{M, \omega}^{2} \leq C\left[\Omega_{1}\left(f, \frac{1}{n}\right)_{M, \omega}\right]^{2} \\
\frac{C}{n^{4}}\left(\sum_{\nu=1}^{n} \nu^{3} \alpha_{\nu}^{2}\right) \leq \alpha_{n+1}^{2}+\left(\Omega_{1}\left(f, \frac{1}{n}\right)_{M, \omega}\right)^{2} \leq C\left[\Omega_{1}\left(f, \frac{1}{n}\right)_{M, \omega}\right]^{2}
\end{gathered}
$$

and hence

$$
\Omega_{1}\left(f, \frac{1}{n}\right)_{M, \omega} \geq \frac{C}{n^{2}}\left(\sum_{\nu=1}^{n} \nu^{3} \alpha_{\nu}^{2}\right)^{1 / 2} .
$$

Proof of Theorem 13. Let $T_{n}$ be a polynomial of class $\mathcal{T}_{n}$ such that $E_{n}(f)_{M, \omega}=$ $\left\|f-T_{n}\right\|_{\langle M\rangle, \omega}$ and we set

菅

$$
\mathcal{U}_{0}(x):=T_{1}(x)-T_{0}(x) ; \mathcal{U}_{\nu}(x):=T_{2^{\nu}}(x)-T_{2^{\nu-1}}(x), \quad \nu=1,2,3, \ldots
$$

Hence

$$
T_{2^{N}}(x)=T_{0}(x)+\sum_{\nu=0}^{N} \mathcal{U}_{\nu}(x), \quad N=0,1,2, \ldots
$$


For a given $\varepsilon>0$, by (1.7) there exists $\eta \in \mathbb{N}$ such that

$$
\sum_{\nu=2^{\eta}}^{\infty} \nu^{\gamma_{1} \alpha-1} E_{\nu}^{\gamma_{1}}(f)_{M, \omega}<\varepsilon
$$

From fractional analog of Bernstein inequality we have

$$
\left\|\mathcal{U}_{\nu}^{(\alpha)}\right\|_{\langle M\rangle, \omega} \leq C 2^{\nu \alpha}\left\|\mathcal{U}_{\nu}\right\|_{\langle M\rangle, \omega} \leq C 2^{\nu \alpha} E_{2^{\nu-1}}(f)_{\langle M\rangle, \omega}, \quad \nu \in \mathbb{N} .
$$

On the other hand it is easily seen that

$$
2^{\nu \alpha} E_{2^{\nu-1}}(f)_{M, \omega} \leq C\left\{\sum_{\mu=2^{\nu-2}+1}^{2^{\nu-1}} \mu^{\gamma \alpha-1} E_{\mu}^{\gamma}(f)_{M, \omega}\right\}^{1 / \gamma_{1}} \quad, \quad \nu=2,3,4, \ldots
$$

For the positive integers satisfying $K<N$

$$
T_{2^{N}}^{(\alpha)}(x)-T_{2^{K}}^{(\alpha)}(x)=\sum_{\nu=K+1}^{N} U_{\nu}^{(\alpha)}(x), \quad x \in \mathbb{T}
$$

and hence if $K, N$ are large enough we obtain from (2.1) that

$$
\begin{aligned}
\left\|T_{2^{N}}^{(\alpha)}-T_{2^{K}}^{(\alpha)}\right\|_{\langle M\rangle, \omega} & \leq \sum_{\nu=K+1}^{N}\left\|\mathcal{U}_{\nu}^{(\alpha)}\right\|_{\langle M\rangle, \omega} \leq C \sum_{\nu=K+1}^{N} 2^{\nu \alpha} E_{2^{\nu-1}}(f)_{M, \omega} \\
& \leq C \sum_{\nu=K+1}^{N}\left\{\sum_{\mu=2^{\nu-2}}^{2^{\nu-1}} \mu^{\gamma \alpha-1} E_{\mu}^{\gamma}(f)_{M, \omega}\right\}^{1 / \gamma} \\
& \leq C\left\{\sum_{\mu=2^{K-1}+1}^{2^{N-1}} \mu^{\gamma \alpha-1} E_{\mu}^{\gamma}(f)_{M, \omega}\right\}^{1 / \gamma} \leq C \varepsilon^{1 / \gamma} .
\end{aligned}
$$

Therefore $\left\{T_{2^{N}}^{(\alpha)}\right\}$ is a Cauchy sequence in $L_{M, \omega}(\mathbb{T})$. Then there exists a $\varphi \in$ $L_{M, \omega}(\mathbb{T})$ satisfying

$$
\left\|T_{2^{N}}^{(\alpha)}-\varphi\right\|_{\langle M\rangle, \omega} \longrightarrow 0, \quad \text { as } N \rightarrow \infty
$$

On the other hand, by the method of proof of Theorem 6 we have

$$
\left\|T_{2^{N}}^{(\alpha)}-f^{(\alpha)}\right\|_{\langle M\rangle, \omega} \longrightarrow 0, \quad \text { as } N \rightarrow \infty .
$$

Then $f^{(\alpha)}=\varphi$ a.e. Therefore $f \in W_{M, \omega}^{\alpha}$. 
We note that

$E_{n}\left(f^{(\alpha)}\right)_{M, \omega} \leq\left\|f^{(\alpha)}-S_{n} f^{(\alpha)}\right\|_{\langle M\rangle, \omega}$

$$
\leq\left\|S_{2^{m+2}} f^{(\alpha)}-S_{n} f^{(\alpha)}\right\|_{\langle M\rangle, \omega}+\left\|\sum_{k=m+2}^{\infty}\left[S_{2^{k+1}} f^{(\alpha)}-S_{2^{k}} f^{(\alpha)}\right]\right\|_{\langle M\rangle, \omega} .
$$

By Lemma 1 we get for $2^{m}<n \leq 2^{m+1}$

$$
\left\|S_{2^{m+2}} f^{(\alpha)}-S_{n} f^{(\alpha)}\right\|_{\langle M\rangle, \omega} \leq C 2^{(m+2) \alpha} E_{n}(f)_{M, \omega} \leq C n^{\alpha} E_{n}(f)_{M, \omega} .
$$

By (1.6) we find

$$
\begin{aligned}
& \| \sum_{k=m+2}^{\infty}\left[S_{2^{k+1}} f^{(\alpha)}-S_{2^{k}} f^{(\alpha)}\right] \|_{\langle M\rangle, \omega} \\
& \leq C\left\|\left\{\sum_{k=m+2}^{\infty}\left|\sum_{\nu=2^{k}+1}^{2^{k+1}} \nu^{\alpha} A_{\nu}\left(x+\frac{\alpha \pi}{2 \nu}, f\right)\right|^{2}\right\}^{1 / 2}\right\|_{\langle M\rangle, \omega}
\end{aligned}
$$

and therefore

$$
\begin{aligned}
& \left\|\sum_{k=m+2}^{\infty}\left[S_{2^{k+1}} f^{(\alpha)}-S_{2^{k}} f^{(\alpha)}\right]\right\|_{\langle M\rangle, \omega} \\
& \text { N } \\
& \leq C\left(\sum_{k=m+2}^{\infty}\left\|\sum_{\nu=2^{k}+1}^{2^{k+1}} \nu^{\alpha} A_{\nu}\left(x+\frac{\alpha \pi}{2 \nu}, f\right)\right\|_{\langle M\rangle, \omega}^{\gamma}\right)^{1 / \gamma} . \\
& \text { Putting } \\
& \delta_{\nu}^{*}:=\sum_{\nu=2^{k}+1}^{2^{k+1}} \nu^{\alpha} A_{\nu}\left(x+\frac{\alpha \pi}{2 \nu}, f\right) \\
& \text { we have } \\
& \left\|\delta_{\nu}^{*}\right\|_{\langle M\rangle, \omega}=\left\|\sum_{\nu=2^{k}+1}^{2^{k+1}} \nu^{\alpha} U_{\nu}(x)\right\|_{M, \omega}
\end{aligned}
$$


where $U_{\nu}(x)=A_{\nu}\left(x+\frac{\alpha \pi}{2 \nu}, f\right)$. Using Abel's transformation we get

$$
\begin{aligned}
\left\|\delta_{\nu}^{*}\right\|_{\langle M\rangle, \omega} \leq & \sum_{\nu=2^{k}+1}^{2^{k+1}-1}\left|\nu^{\alpha}-(\nu+1)^{\alpha}\right|\left\|\sum_{l=2^{k}+1}^{\nu} U_{l}(x)\right\|_{\langle M\rangle, \omega} \\
& +\left|\left(2^{k+1}\right)^{\alpha}\right|\left\|\sum_{l=2^{k}+1}^{2^{k+1}-1} U_{l}(x)\right\|_{\langle M\rangle, \omega} .
\end{aligned}
$$

Since

$$
\begin{aligned}
A_{\nu}\left(x+\frac{\alpha \pi}{2 \nu}, f\right)= & a_{\nu}(f) \cos \left(\nu x+\frac{\alpha \pi}{2}\right)+b_{\nu}(f) \sin \left(\nu x+\frac{\alpha \pi}{2}\right) \\
= & a_{\nu}(f)\left[\cos \nu x \cos \frac{\alpha \pi}{2}-\sin \nu x \sin \frac{\alpha \pi}{2}\right] \\
& +b_{\nu}(f)\left[\sin \nu x \cos \frac{\alpha \pi}{2}+\cos \nu x \sin \frac{\alpha \pi}{2}\right] \\
= & \cos \frac{\alpha \pi}{2}\left[a_{\nu}(f) \cos \nu x+b_{\nu}(f) \sin \nu x\right] \\
& +\sin \frac{\alpha \pi}{2}\left[b_{\nu}(f) \cos \nu x-a_{\nu}(f) \sin \nu x\right] \\
= & \cos \frac{\alpha \pi}{2} A_{\nu}(x, f)-\sin \frac{\alpha \pi}{2} A_{\nu}(x, \tilde{f})
\end{aligned}
$$

we have that, for $2^{k}+1 \leq \nu \leq 2^{k+1},(k \in \mathbb{N})$

$$
\begin{aligned}
\left\|\sum_{l=2^{k}+1}^{\nu} U_{l}(x)\right\|_{\langle M\rangle, \omega} & =\left\|\sum_{l=2^{k}+1}^{\nu} A_{l}\left(x+\frac{\alpha \pi}{2 l}, f\right)\right\|_{\langle M\rangle, \omega} \\
& =\left\|\sum_{l=2^{k}+1}^{\nu}\left(\cos \frac{\alpha \pi}{2} A_{l}(x, f)-\sin \frac{\alpha \pi}{2} A_{l}(x, \tilde{f})\right)\right\|_{\langle M\rangle, \omega} \\
& \leq\left\|\sum_{l=2^{k}+1}^{\nu} A_{l}(x, f)\right\|_{\langle M\rangle, \omega}+\left\|\sum_{l=2^{k}+1}^{\nu} A_{l}(x, \tilde{f})\right\|_{\langle M\rangle, \omega} \\
& \leq C\left(E_{2^{k}}(f)_{M, \omega}+E_{2^{k}}(\tilde{f})_{M, \omega}\right) \leq C E_{2^{k}}(f)_{M, \omega} .
\end{aligned}
$$

In the last inequality we used (1) of Remark 2. Using

$$
(\nu+1)^{\alpha}-\nu^{\alpha} \leq \begin{cases}\alpha(\nu+1)^{\alpha-1} & , \alpha \geq 1 \\ \alpha \nu^{\alpha-1} & , 0 \leq \alpha<1\end{cases}
$$

we obtain

$$
\left\|\delta_{\nu}^{*}\right\|_{\langle M\rangle, \omega} \leq C 2^{k \alpha} E_{2^{k-1}}(f)_{M, \omega}
$$


Therefore

$$
\begin{aligned}
\left\|_{k=m+2}^{\infty}\left[S_{2^{k+1}} f^{(\alpha)}-S_{2^{k}} f^{(\alpha)}\right]\right\|_{\langle M\rangle, \omega} & \leq C\left\{\sum_{k=m+2}^{\infty} 2^{k \alpha \gamma} E_{2^{k}-1}^{\gamma}(f)_{M, \omega}\right\}^{1 / \gamma} \\
& \leq C\left\{\sum_{\nu=n+1}^{\infty} \nu^{\gamma \alpha-1} E_{\nu-1}^{\gamma}(f)_{M, \omega}\right\}^{1 / \gamma}
\end{aligned}
$$

and using (2.2)-(2.4) Theorem 13 is proved.

Acknowledgement. The author indebted to referees for valuable suggestions and comments. Part of this research was written when the author was staying at the Centre de Recerca Matemàtica, Group Harmonic analysis and Approximation Theory.

\section{REFERENCES}

[A] R. Akgün, Sharp Jackson and converse theorems of trigonometric approximation in weighted Lebesgue spaces, Proc. A. Razmadze Math. Inst., 152 (2010), 1-18.

[AI] R. Akgün and D. M. Israfilov, Simultaneous and converse approximation theorems in weighted Orlicz spaces, Bull. Belg. Math. Soc. Simon Stevin, 17 (2010), no. 1, 13-28.

[AI1] R. Akgün and D. M. Israfilov, Approximation in weighted Orlicz spaces, Math. Slovaca, 61 (2011) no. 4, 601-618.

[BS] N. K. Bari and S. B. Stechkin, Best approximations and differential properties of two conjugate functions, (Russian), Trudy Moskov. Mat. Obsch., 5 (1956), 483-522.

[BDGS] P. L. Butzer; H. Dyckhoff; E. Görlich and R. L. Stens, Best trigonometric approximation, fractional order derivatives and Lipschitz classes, Canad. J. Math., 29 (1977), no. $4,781-793$.

[CCL] L. Y. Chan; Y.-M. Chen and M. C. Liu, Some properties of asymptotic functions, Studia Math., 67 (1980), no. 1, 65-72.

[Che] Yung-Ming Chen, Theorems of asymptotic approximation, Math. Ann., 140 (1960), 360-407.

v [Che1] Yung-Ming Chen, On two-functional spaces, Studia Math., 24 (1964), 61-88.

[CGMP] G. P.Curbera; J. García-Cuerva; J. M. Martell and C. Pérez, Extrapolation with weights, rearrangement-invariant function spaces, modular inequalities and applications to singular integrals, Adv. Math., 203 (2006), no. 1, 256-318.

[Gadj] E. A. Gadjieva, Investigation of the properties of functions with quasimonotone Fourier coefficients in generalized Nikolskii-Besov spaces, author's summary of dissertation, Tbilisi, 1986, (In Russian).

[GI A. Guven and D. M. Israfilov, Improved inverse theorems in weighted Lebesgue and Smirnov spaces, Bull. Belg. Math. Soc. Simon Stevin, 14 (2007), no. 4, 681-692.

[HKT] C. Hao; A. Kamińska and N. Tomczak-Jaegermann, Orlicz spaces with convexity or concavity constant one, J. Math. Anal. Appl., 320 (2006), no. 1, 303-321.

[IG] D. M. Israfilov and A. Guven, Approximation by trigonometric polynomials in weighted Orlicz spaces, Studia Math., 174 (2006), no. 2, 147-168.

[Kh] M. Khabazi, The mean convergence of trigonometric Fourier series in weighted Orlicz classes, Proc. A. Razmadze Math. Inst., 129 (2002), 65-75. 
[KK] V. Kokilashvili and M. Krbec, Weighted inequalities in Lorentz and Orlicz spaces, World Scientific Publishing Co., Inc., River Edge, NJ, 1991.

[KY1] V. Kokilashvili and Y. E. Yildirir, The estimation of high order generalized modulus of continuity in $L_{\omega}^{p}$, Proc. A. Razmadze Math. Inst., 143 (2007), 135-137.

$[\mathrm{KY}] \quad$ V. Kokilashvili and Y. E. Yildirir, On the approximation in weighted Lebesgue spaces, Proc. A. Razmadze Math. Inst., 143 (2007), 103-113.

[KY2] V. Kokilashvili and Y. E. Yildirir, On the approximation by trigonometric polynomials in weighted Lorentz spaces, J. Funct. Spaces Appl., 8 (2010), no. 1, 67-86.

[Kon] A. A. Konyushkov, Best approximations by trigonometric polynomials and Fourier coefficients, (Russian), Mat. Sb. N.S. 44(86) (1958), 53-84.

$[\mathrm{Ku}] \quad$ D. S. Kurtz, Littlewood-Paley and multiplier theorems on weighted $L_{p}$ spaces, Trans. Amer. Math. Soc., 259 (1980), no. 1, 235-254.

[Ky1] N. X. Ky, On approximation by trigonometric polynomials in $L_{u}^{p}$-spaces, Studia Sci. Math. Hungar., 28 (1993), no. 1-2, 183-188.

[Ky2] N. X. Ky, Modulus of mean smoothness and approximation with $A_{p}$-weights, Ann. Univ. Sci. Budapest. Eötvös Sect. Math., 40 (1997), 37-48.

[Ky3] N. X. Ky, An Alexits's lemma and its applications in approximation theory, Functions, series, operators (Budapest, 1999), 287-296, János Bolyai Math. Soc., Budapest, 2002.

[PS] M. K. Potapov and B. V. Simonov, On the interrelation of the generalized BesovNikolskii and Weyl-Nikolskii classes of functions, Anal. Math., 22 (1996), no. 4, 299316.

[ST] B. V. Simonov and S. Yu. Tikhonov, Embedding theorems in the constructive theory of approximations, (Russian), Mat. Sb. 199 (2008), no. 9, 107-148; translation in Sb. Math., 199 (2008), no. 9-10, 1367-1407.

[S] S. B. Stechkin, On the absolute convergence of Fourier series, Izv. Akad. Nauk. SSSR. Ser. Mat., 17 (1953), 499-512, (In Russian).

[Tab] R. Taberski, Differences, moduli and derivatives of fractional orders, Comment. Math. Prace Mat., 19 (1976/77), no. 2, 389-400.

[TiM] M. F. Timan, Inverse theorems of the constructive theory of functions in $L_{p}$ spaces $(1 \leq p \leq \infty)$, (Russian), Mat. Sb. N.S. 46(88) (1958), 125-132.

[TiM1] M. F. Timan, On Jackson's theorem in $L_{p}$ spaces, (Russian), Ukrain. Mat. Z., 18 (1966), no. 1, 134-137.

[YI $\quad$ Y. E. Yildirir and D. M. Israfilov, Approximation theorems in weighted Lorentz spaces, Carpathian J. Math., 26 (2010), no. 1, 108-119.

[ZYG] A. Zygmund, Trigonometric series, 2nd ed., Vols. I and II, Cambridge University Press, New York, 1959.

BALIKESIR UNIVERSITY

Faculty of Arts and Sciences

DePARTMENT OF MATHEMATiCS

10145, ÇAĞIŞ YERLEŞKESI

BALIKESIR, TURKEY

E-mail address: rakgun@balikesir.edu.tr, 



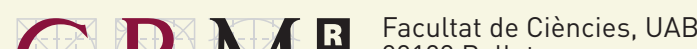

08193 Bellaterra

Barcelona, Spain

CENTRE DE RECERCA MATEMÀTICA WWW.crm.cat 